\title{
Integrated imaging and vision techniques for industrial inspection: a special issue on machine vision and applications
}

\author{
Zheng Liu • Hiroyuki Ukida • Pradeep Ramuhalli • \\ David S. Forsyth
}

Published online: 5 June 2010

(C) Springer-Verlag 2010

\begin{abstract}
Imaging- and vision-based techniques play an important role in industrial inspection. The sophistication of the techniques assures high-quality performance of the manufacturing process through precise positioning, online monitoring, and realtime classification. Advanced systems incorporating multiple imaging and/or vision modalities provide robust solutions to complex situations and problems in industrial applications. A diverse range of industries, including aerospace, automotive, electronics, pharmaceutical, biomedical, semiconductor, and food/beverage, etc., have benefited from recent advances in multi-modal imaging, data fusion, and computer vision technologies. Many of the open problems in this context are in the general area of image analysis methodologies (preferably in an automated fashion). The purpose of this special issue is to highlight recent advances and demonstrate the successful applications of integrated imaging and vision technologies in industrial inspection.
\end{abstract}

\section{Z. Liu $(\bowtie)$}

NRC Institute for Research in Construction, 1200 Montreal Road, Ottawa, ON K1A 0R6, Canada

e-mail: zheng.liu@nrc-cnrc.gc.ca

\section{H. Ukida}

Department of Mechanical Engineering,

The University of Tokushima, 2-1 Minamijosanjima-cho,

Tokushima, 770-8506, Japan

e-mail: ukida@me.tokushima-u.ac.jp

P. Ramuhalli

Pacific Northwest National Laboratory, MSIN K5-26,

902 Battelle Boulevard, P.O. Box 999, Richland, WA 99352, USA

e-mail: pradeep.ramuhalli@pnl.gov

D. S. Forsyth

NDE Division, Texas Research International Inc. Austin,

9063 Bee Caves Road, Austin, TX 78733, USA

e-mail: dforsyth@tri-austin.com
Practical applications may require innovative use of multiple techniques to achieve an optimal solution.

There are eight papers in this special issue. These papers advance the methodologies for image acquisition, processing, interpretation, and analysis for varied industrial applications. All the papers are original and tackle different problems in industrial inspection, often using more than one technique to acquire, process and interpret the image data.

A crucial issue in the analysis of multi-modal imagingand vision-based data is image registration, to ensure that measurements are spatially and temporally from the same object regions. Breitenreicher et al. [1] propose a probabilistic objective function for the registration of rigid point sets without requiring explicit correspondence. The major advantage of this method is its robustness with the presence of spatially outlying structures. The estimation of object pose is further implemented by computing the gradient flow directly on the related manifold. This research is useful to the application of 3D vision guided manipulation of components, like bin picking systems.

A good set of features are crucial to the performance of a classifier in a surface inspection system, where a binary decision, i.e. accept/reject, is necessary. The inspection of bearing, eggs, and machined castings may fall into this type of application. Eitzinger et al. [2] address these issues with image processing and classification for such applications in their paper. The authors implemented an adaptive feature calculation, feature pre-processing, and image classification and carried out a quantitative assessment of the performance of these adaptive operations.

Another paper presented by Raiser et al. [3] from the same research group studies the impact of varied object extraction approaches for image classification in surface inspection. Various methods to group the pixels representing potential defect areas were investigated, including classical image 
processing techniques and data clustering approaches. The challenge is to group non-connected or widespread objects. The image classifiers were trained with the features derived from the extracted objects. A density-based clustering algorithm named BDSCAN was shown to outperform the other methods in the tests. This clustering approach is further optimized to process a large number of data points.

The problem of image acquisition and synthesis is addressed in the paper by Tabata et al. [4]. The paper describes a surface image synthesis algorithm for the highspeed imaging and inspection of moving and spinning objects. The paper describes an FPGA-based high-speed vision system to detect printing defects or recognize bar codes on beverage cans that are moving and spinning simultaneously. A surface image synthesis of the spinning cans based on the principle of image mosaic is proposed. A commercially available high-speed area scan camera was used to capture the spinning cans at the frame rate of 1,000 fps. With this implementation, the authors also provided a solution to compensate the influence of diffuse reflection due to surface curvature and estimate the dimensional errors, such as image distortion and quantization errors in the synthesized image. This technique has great potential for industrial applications, where inspection of moving and spinning objects is required.

Martin et al. [5] from the Industrial Automation Institute from Spain have applied multiple inspection techniques for the detection of residual oxide scale on a cold stainless steel strip. In the stainless steel manufacturing process, residual oxide scale on stainless steel surfaces must be detected to avoid corrosion and for quality control purposes. The authors described three different imaging systems applied to address this problem. The first system employed a commercial laser sensor with a dynamic threshold algorithm. This system can detect defects up to 50 microns at a moving speed of $1 \mathrm{~m} / \mathrm{s}$. The second technique is based on optical emission spectroscopy (OES). The inspection is accomplished by using OES to analyze the plasma generated by volatilizing a small amount of stainless steel surface with a very short pulse of highenergy ultraviolet laser light. The third approach employed a machine vision system to inspect the surface with laser diode diffuse illumination. Two illumination modes were implemented in this system: diffuse coaxial lighting and diffuse bright-field lighting. The results indicate that these three techniques are particularly well-suited to detecting residual oxide scale on stainless steel surfaces.

Flank wear of microdrills used in the electronic manufacturing industry is a major issue, and detection and monitoring of flank wear is necessary for timely replacement and maintenance activities to ensure finished product quality. The measurement of flank wear can be carried out with automatic optical inspection (AOI). To detect defects, the cutting plane needs to be identified from the acquired microdrill bit image. The challenge is that the cutting plane is stained by smear- ing during the manufacturing process and cannot be easily segmented with conventional techniques. Duan et al. [6] propose the use of the level set method to tackle this problem. They further characterize the flank wear with a projection profile based method and define an index, namely "end wear length", to assess drill life.

Inspecting transmission power line remotely from unmanned aerial vehicles (UAVs) with optical sensor becomes a challenging task especially when the background in the acquired aerial imagery is cluttered. In other words, the automatic power line detection is not easy to achieve due to the flight height and resolution of the camera. $\mathrm{Li}$ et al. [7] propose a knowledge-based technique, in which a filter based on pulse coupled neural network is applied to remove the background noise and the Hough transform together with a knowledge-based line clustering strategy used to refine the power line detection. The proposed approach increased the accuracy of power line detection in a complex environment. Thus, this technique provides an efficient tool for the maintenance and monitoring of power lines.

Nakazawa et al. [8] presented their studies on materials' deformation/fracture (D/F) characteristics with nano-order 3D-CT images. Their approach is able to analyze the nanoscale $\mathrm{D} / \mathrm{F}$ characteristics inside materials. Internal structures were extracted from the 3D-CT slice images with discriminant analysis and a threshold operation. A stratified method was proposed to match impurity particles with deformation by the authors. Finally, 3D strain distribution maps were built to evaluate $\mathrm{D} / \mathrm{F}$ characteristics.

The novelty and originality of the papers published in this special issue contribute significantly to the research of vision system and will promote the use of modern machine vision techniques in the field of industrial inspection.

Acknowledgments The guest editors of this special issue would like to thank all anonymous reviewers for their time and efforts in reviewing the submissions.

\section{References}

1. Breitenreicher, D., Schnorr, C.: Robust 3D object registration without explicit correspondence using geometic integration. Mach. Vis. Appl. (2009). doi:10.1007/s00138-009-0227-6

2. Eitzinger, C., Heidl, W., Lughofer, E., Raiser, S., Smith, J.E., Tahir, M.A., Sannen, D., Van Brussel, H.: Assessment of the influence of adaptive components in trainable surface inspection systems. Mach. Vis. Appl. (2009). doi:10.1007/s00138-009-0211-1

3. Raiser, S., Lughofer, E.D., Eitzinger, C., Smith, J.E.: Impact of object extraction methods on classification performance in surface inspection systems. Mach. Vis. Appl. (2009). doi:10.1007/s00138009-0205-z

4. Tabata, T., Komuro, T., Ishidawa, M.: Surface image synthesis of moving spinning cans using a 1000 -fps area scan camera. Mach. Vis. Appl. (2010). doi:10.1007/s00138-010-0247-2 
5. Martin, D., Guinea, D.M., Garcia-Alegre, M.C., Villanueva, E., Guinea, D.: Multi-modal defect detection of residual oxide scale on a cold stainless steel strip. Mach. Vis. Appl. (2010). doi:10.1007/ s00138-010-0260-5

6. Duan, G., Chen, Y.-W., Sukekawa, T.: Automatic optical flank wear measurement of microdrills using level set for cutting plane segmentation. Mach. Vis. Appl. (2010). doi:10.1007/s00138-010$0259-\mathrm{y}$
7. Li, Z., Liu, Y., Walker, R., Haywar, R., Zhang, J.: Towards automatic power line detection for UAV surveillance system using pulse coupled neural filter and an improved Hough transform. Mach. Vis. Appl. (2009). doi:10.1007/s00138-009-0206-y

8. Nakazawa, M., Kobayashi, M., Toda, H., Aoki, Y.: Proposal of a method to analyze 3D deformation/fracture characteristics inside materials based on a stratified matching approach. Mach. Vis. Appl. (2010). doi:10.1007/s00138-009-0242-7 